\title{
A Constraint Learning Algorithm for Blind Source Separation
}

\author{
Kenji Nakayama Akihiro Hirano Motoki Nitta \\ Dept. of Elec and Comp Eng., Kanazawa Univ., 920-8667, Japan \\ E-mail:nakayama@t.kanazawa-u.ac.jp
}

\begin{abstract}
In Jutten's blind separation algorithm, symmetrical distribution and statistical independence of the signal sources are assumed. When they are not satisfied, the learning process becomes unstable. In order to avoid the unstable behavior, two stabilization methods are proposed. Since large samples easily disturb symmetrical distribution, the outputs of the separation process with large amplitude are detected, and the learning is skipped. Imbalance of the signal source powers affects statistical independence. It is estimated by the cross-correlation of the observed signals. When the cross-correlation is high, the correction term by the Jutten's algorithm becomes wrong. Therefore, adjusting the weights in the separation process is skipped. Computer simulation using many kinds of signal sources demonstrates the signal sources with asymmetrical distribution and imbalanced power are well separated.

Keywords: Blind separation, Stability, Probability density function, Correlation coefficient, Speech
\end{abstract}

\section{Introduction}

Recently, many kinds of information are transmitted and processed. At the same time, high quality is required. For this reason, signal processing including noise cancelation, echo cancelation, equalization of transmission line, restoration of signals have been becoming very important technology. In some cases, we do not have enough information about signals and interference. Furthermore, their mixing process and transmission processes are not well known. Under these situations, blind separation methods using statistical property of the signal sources have become important [1]-[5].

Jutten et all proposed a blind separation algorithm based on statistical independence and symmetrical distribution of the signal sources [6]-[8]. The learning algorithm is derived assuming approximate convergence. That is, some of outputs are expected to be similar to the signal sources. However, this causes unstable behavior during early stage of the learning process. Furthermore, when some signal source level is dominant, it is difficult to make the outputs statistically independent.

In this paper, two stabilization methods are proposed. The first method corresponds to symmetrical distribution, and the second one deals with imbalance of the signal source levels. In any cases, the situations, where the Jutten's learning algorithm is unstable, are detected, and the adaptation of parameters is controlled. Many kinds of combinations of signal sources are examined through computer simulation, in order to confirm usefulness of the proposed stabilization methods.

\section{Jutten's Blind Separation}

\subsection{Network Structure}

Figure 1 shows a blind separation model proposed by Jutten et all [6]. The number of the signal sources, the sensors and the outputs are all the same. The signal sources $s_{i}(n), i=1,2, \ldots, N$ are linearly combined using unknown weights $a_{j i}$, and are sensed at $\mathrm{N}$ points, resulting in $x_{j}(n)$. A general form is

$$
x_{j}(n)=\sum_{i=1}^{N} a_{j i} s_{i}(n)
$$

The output of the separation block $y_{k}(n)$ is given by

$$
y_{j}(n)=x_{j}(n)-\sum_{\substack{k=1 \\ \neq j}}^{N} c_{j k} y_{k}(n)
$$


This relation is expressed using vectors and matrices in the case of $\mathrm{N}=2$ as follows:

$$
\begin{aligned}
\boldsymbol{x}(n) & =\boldsymbol{A s}(n) & \\
\boldsymbol{y}(n) & =\boldsymbol{x}(n)-\boldsymbol{C y}(n) & \\
\boldsymbol{A} & =\left[\begin{array}{ll}
a_{11} & a_{12} \\
a_{21} & a_{22}
\end{array}\right] & \boldsymbol{C}=\left[\begin{array}{ll}
c_{11} & c_{12} \\
c_{21} & c_{22}
\end{array}\right]
\end{aligned}
$$

$\boldsymbol{A}$ is an unknown mixing matrix. From these expressions, a relation between the signal sources and the separation outputs becomes

$$
\boldsymbol{y}(n)=(\boldsymbol{I}+\boldsymbol{C})^{-1} \boldsymbol{x}(n)=(\boldsymbol{I}+\boldsymbol{C})^{-1} \boldsymbol{A} \boldsymbol{s}(n)
$$

The following matrix can be regarded as a separation matrix.

$$
\boldsymbol{W}=(\boldsymbol{I}+\boldsymbol{C})^{-1}
$$

In order to evaluate separation performance, the following matrix is defined.

$$
\boldsymbol{P}=\boldsymbol{W} \boldsymbol{A}
$$

If $\boldsymbol{P}$ takes the next forms, the signals $s_{1}$ and $s_{2}$ are completely separated at ether $y_{1}$ or $y_{2}$.

$$
\boldsymbol{P}=\left[\begin{array}{cc}
p_{11} & 0 \\
0 & p_{22}
\end{array}\right] \quad \text { or } \quad\left[\begin{array}{cc}
0 & p_{12} \\
p_{21} & 0
\end{array}\right]
$$

\subsection{Learning Algorithm}

Error Function:

It is assumed that the separation block approaches the optimum solution, then $N-1$ outputs are already proportional to one of the signal sources. Only one output has not reached to the signal source. Let $y_{l}(n)$ be this output. From Eq.(2),

$$
y_{l}(n)=x_{l}(n)-\sum_{\substack{k=1 \\ \neq l}}^{N} c_{l k} y_{k}(n)
$$

Here, $y_{k}$ is already $a_{k k} s_{k}(n)$. Furthermore, $x_{l}(n)$ is replaced by Eq.(1).

$$
y_{l}(n)=\sum_{\substack{k=1 \\ \neq l}}^{N}\left(a_{l k}-c_{l k} a_{k k}\right) s_{k}(n)+a_{l l} s_{l}(n)
$$

The mean squared $y_{l}(n)$ becomes

$$
J_{l}=E\left[y_{l}^{2}(n)\right]=\sum_{\substack{k=1 \\ \neq l}}^{N}\left(a_{l k}-c_{l k} a_{k k}\right)^{2} E\left[s_{k}^{2}(n)\right]+a_{l l}^{2} E\left[s_{l}^{2}(n)\right]
$$

In the above derivation, $s_{k}(n)$ are assumed to be independent to each other. Furthermore, it is assumed that mean of $s_{i}(n)$ is zero, then mean of $y_{k}(n)$ are also zero.

In $E\left[y_{l}^{2}(n)\right]$, only $\left(a_{l k}-c_{l k} a_{k k}\right)^{2}$ can be controlled by the separation block. It can be zero for $c_{l k}=a_{l k} / a_{k k}$. Thus, the optimum solution can be obtained employing $J_{l}$ as a cost function.

Gradient Method:

Instead of $J_{l}=E\left[y_{l}^{2}(n)\right]$, the instantaneous value $y_{l}^{2}(n)$ is used as a cost function, like Least Mean Square (LMS) algorithm [9]. The partial derivative becomes [6]

$$
\frac{\partial y_{l}^{2}}{\partial c_{l k}}=-2 q_{l l} y_{l}(n) y_{k}(n)
$$


$q_{l l}$ is the $(l, l)$ th element of $(\boldsymbol{I}+\boldsymbol{C})^{-1}$. An update equation becomes

$$
c_{l k}(n+1)=c_{l k}(n)+\eta q_{l l} y_{l}(n) y_{k}(n), \quad 0<\eta: \text { stepsize }
$$

Assumptions of Signal Source Properties:

Assumption 1: The signal sources are statistically independent to each other.

Assumption 2: Samples of the signal sources are symmetrically distributed, that is their probability density function (pdf) are even.

The update equation Eq.(14) is further replaced by the next taking the above conditions into account.

$$
c_{l k}(n+1)=c_{l k}(n)+\eta f\left(y_{l}(n)\right) g\left(y_{k}(n)\right)
$$

Since both the mixing and the separation processes are linear operations, $y_{k}(n)$ is a linear combination of $s_{i}(n)$, then they have also an even pdf. Furthermore, if the step size $\eta$ is set to a small number, the update by Eq.(15) is equivalent to calculating the mean of $f\left(y_{l}(n)\right) g\left(y_{k}(n)\right)$, thus,

$$
\Delta c_{l k}(n) \propto E\left[f\left(y_{l}(n)\right) g\left(y_{k}(n)\right)\right]
$$

As $y_{k}(n)$ approah to $s_{i}(n)$, they can be statistically independent to each other, then

$$
E\left[f\left(y_{l}(n)\right) g\left(y_{k}(n)\right)\right]=E\left[f\left(y_{l}(n)\right)\right] E\left[g\left(y_{k}(n)\right)\right]
$$

Furthermore, by using odd functions for $f()$ and $g()$,

$$
E\left[f\left(y_{l}(n)\right)\right]=0, \quad E\left[g\left(y_{k}(n)\right)\right]=0
$$

Here mean of $s_{i}(n)$, that is of $y_{k}(n)$ is assumed to be zero. If the mean is not actually zero, it can be estimated and will be subtracted from $y_{k}(n)$. Different odd functions are used for $f()$ and $g()$. If they are the same and the initial guess of $c_{k l}$ are zero, the correction terms are always the same, and all $c_{k l}$ becomes the same.

From these discussions, by decreasing the correction term $\eta f\left(y_{k}(n)\right) g\left(y_{l}(n)\right)$, independence among the outputs $y_{k}(n)$ can be guaranteed.

\section{Stabilization of Learning Process}

\subsection{Even Probability Density Function}

\section{Reason for Unstable Behavior:}

Jutten's algorithm assume the even pdf for $s_{i}(n)$, furthermore, $y_{k}(n)$ are close to $s_{i}(n)$. However, during early stage of the learning process, these assumptions are not guaranteed. Although the even pdf can be assumed, it is not valid for a small number of samples. Large samples easily disturb the even pdf condition. Stabilization: Method 1:

In order to avoid disturbance by large samples, they are detected and the learning of $c_{l k}(n)$ is skipped. First, the variance $\sigma_{y_{k}}^{2}$ is estimated using the observed data as follows:

$$
\begin{aligned}
\sigma_{y_{k}}^{2}(n) & =(1-\alpha) \sigma_{y_{k}}^{2}(n-1)+\alpha\left(y_{k}(n)-\overline{y_{k}}(n)\right)^{2} \\
\overline{y_{k}}(n) & =(1-\alpha) \overline{y_{k}}(n-1)+\alpha y_{k}(n), \quad 0<\alpha \ll 1
\end{aligned}
$$

If one of $\left|y_{k}(n)\right|$ exceeds some level, the learning is skipped.

$$
\begin{aligned}
\text { If }\left|y_{k}(n)\right| & >\theta_{L} \sigma_{y_{k}}(n), \text { then } c_{l k}(n+1)=c_{l k}(n), \\
\text { otherwise } \quad c_{l k}(n+1) & =c_{l k}(n)+\eta f\left(y_{l}(n)\right) g\left(y_{k}(n)\right) .
\end{aligned}
$$




\subsection{Imbalance of Signal Source Powers}

\section{Silent Intervals:}

When the signal sources are voices, silent intervals are included. In this interval, the outputs $y_{k}(n)$ have strong correlation. For example, in the case of two signal sources, the observed data are expressed by

$$
\begin{aligned}
& x_{1}(n)=a_{11} s_{1}(n)+a_{12} s_{2}(n) \\
& x_{2}(n)=a_{21} s_{1}(n)+a_{22} s_{2}(n)
\end{aligned}
$$

In the silent interval, where $s_{1}(n)=0, x_{1}(n)$ and $x_{2}(n)$ include only $s_{2}(n)$, then $y_{1}(n)$ and $y_{2}(n)$ also contain only $s_{2}(n)$. The outputs $y_{k}(n)$ cannot be statistically independent. In this interval, if the weights $c_{l k}$ are updated by Eq.(15), they will diverge. Because the solution does not exists along the direction, in which the correction term $f\left(y_{k}(n)\right) g\left(y_{l}(n)\right)$ will decrease. In this interval, even though the separation is complete, that is Eq. (9) is held, $f\left(y_{k}(n)\right) g\left(y_{l}(n)\right)$ cannot be zero. For this reason, the adaptation in this interval causes divergence. This problem will occurs not only in the silent intervals but also for imbalance of signal power. Detection of Signal Power Imbalance:

The following cross-correlation is used for detecting signal power imbalance.

$$
\begin{aligned}
\rho_{x_{1} x_{2}} & =\frac{\sigma_{x_{1} x_{2}}}{\sigma_{x_{1}} \sigma_{x_{2}}} \\
\sigma_{x_{1} x_{2}} & =E\left[\left(x_{1}(n)-\overline{x_{1}}(n)\right)\left(x_{2}(n)-\overline{x_{2}}(n)\right)\right] \\
\sigma_{x_{1}}^{2} & =E\left[\left(x_{1}(n)-\overline{x_{1}}(n)\right)^{2}\right] \\
\sigma_{x_{2}}^{2} & =E\left[\left(x_{2}(n)-\overline{x_{2}}(n)\right)^{2}\right]
\end{aligned}
$$

$\overline{x_{i}}(n)$ is mean of $x_{i}(n)$, which is iteratively calculated. This cross-correlation $\rho_{x_{1} x_{2}}$ is unity in the silent interval, and also takes a large value close to unity in the case of $\sigma_{s_{1}} \ll \sigma_{s_{2}}$ or $\sigma_{s_{1}} \gg \sigma_{s_{2}}$.

Stabilization: Method2:

Using the cross-correlation $\rho_{x_{1} x_{2}}$, the intervals, where the signal powers are imbalance, are detected. For high cross-correlation, the learning process is skipped.

$$
\begin{aligned}
\text { If } \rho_{x_{1} x_{2}} & >\theta_{C}, \quad \text { then } \quad c_{l k}(n+1)=c_{l k}(n), \\
\text { otherwise } \quad c_{l k}(n+1) & =c_{l k}(n)+\eta f\left(y_{l}(n)\right) g\left(y_{k}(n)\right) .
\end{aligned}
$$

\section{Simulation and Discussions}

\subsection{Simulation Conditions}

The number of the signal sources is two. The unknown mixing process and the odd functions are

$$
\begin{aligned}
\boldsymbol{A} & =\left[\begin{array}{cc}
1 & 0.6 \\
0.3 & 1
\end{array}\right] \\
f(y) & =y^{3} \quad g(y)=y
\end{aligned}
$$

\subsection{Non-even pdf of Signal Sources}

$s_{1}(n)$ follows Poisson distribution, with mean $=1$ and variance $=1$. This distribution is further shifted so as to make the mean zero. $s_{2}(n)$ follows uniform distribution or normal distribution, with mean $=0$, variance $=1$. The threshold $\theta_{L}$ used for detecting large samples, is set to $\theta_{L}=2.5$. The separation is evaluated by $\boldsymbol{P}$ defined by Eq.(8). Tabel 1 shows the simulation results. PU-NL means Poisson and uniform distributions are used, and the learning of $c_{l k}$ is always carried out. PN-L means Poisson and normal distributions are used, and the learning of $c_{l k}$ is skipped for the large samples, which exceed $\theta_{L} \sigma_{y_{k}}(n)$. Since the uniform distribution does not generate large samples, the ordinary Jutten's algorithm (PU-NL) works well. However, the normal distribution includes large samples with low probability. The assumption of symmetrical distribution is disturbed in early stage. As a result, the conventional (PN-NL) does not work well. On the contrary, the proposed constraint algorithm (PN-L) can separate the signal sources very well. 


\subsection{Signal Power Imbalance}

Voice signal and white noise are used for $s_{1}(n)$ and $s_{2}(n)$, respectively. Figure 2 shows the waveform of the voice and the white noise, and the cross-correlation $\rho_{x_{2} x_{2}}$. In the figure of $\rho_{x_{1} x_{2}}$, the horizontal line indicates $\theta_{C}=0.8$. In the silent intervals, where the voice level is very small, $\rho_{x_{1} x_{2}}$ is close to unity. On the other hand, when the voice has high level, $\rho_{x_{1} x_{2}}$ is reduced from unity. It is possible to distinguish these states. Table 2 shows the numerical data of $\boldsymbol{P}$. In this case, both constraints (LC), that is, the amplitude limitation (L) and the cross-correlation (C), are necessary to achieve good separation performance.

\subsection{Correlated Signal Sources}

Combinations of voice and voice, which have some correlation, are taken into account. The thresholds are set so that $\theta_{L}=1$ and $\theta_{C}=0.8$. Figure 3 shows the waveform of the voices and $\rho_{x_{1} x_{2}}$. At the silent intervals, $\rho_{x_{1} x_{2}}$ takes value close to unity. The learning intervals, where $\rho_{x_{1} x_{2}}<\theta_{C}$ is satisfied, is 22.8 percentages of the entire interval. The resulting $\boldsymbol{P}$ is

$$
\boldsymbol{P}=\left[\begin{array}{cc}
1.074 & 0.111 \\
-0.145 & 0.954
\end{array}\right]
$$

Although the cross terms $p_{12}=0.111$ and $p_{21}=-0.145$ are slightly increased, good separation is obtained.

\section{Conclutions}

Stabilization methods for Jutten's algorithm have been proposed. In order to guarantee symmetrical distribution of the signal sources, large samples of the separation outputs are detected and the learning of the weights is skipped. Power imbalance and some correlations among the signal sources may break independence among the separation outputs, and cause divergence in the learning process. This situation is detected using the cross-correlation among the observed data, and the learning process is controlled. Many kinds of combinations of the signal sources have been examined though computer simulation. The proposed method demonstrated good separation compared with the ordinary Jutten's algorithm.

\section{References}

[1] C.Jutten, J.Herault and A.Guerin, "IIN.C.A: An independent components analyzer based on an adaptive neuromimetic network", in: J.Degmongeot, T.Herve, V.Raille and C.Roche, eds., Artificial Intelligence and Cognitive Science, Manhester Univ. Press, Manchester, 1988.

[2] C.Jutten and J.Herault, "Analog implementation of permanent unsupervised learning algorithm", Proc. NATO Advanced Research Workshop on Neurocomputing, Les Arcs, France, pp.145-152, Feb.27-March 3, 1989.

[3] P.Comon, "Separation of stochastic process whose linesr mixtureis observed", Proc. ONR- NSF-IEEE Workshop on Higher Spectral Analysis Vail, Colorado, pp.174-179, June 28-30, 1989.

[4] P.Comon,"Separation of sources using higher-order cumulants", SPIE conference, Vol.1152, Advanced Algorithms and Architectures for Signal Processing IV, San Diego, California, pp. 170-181, August 6-11, 1989.

[5] J.F.Cardoso, "Eigen structure of the 4th order cumulant tensor with application to the blind source separation problem", ICASSP Proc. pp. 2655-1658.

[6] C.Jutten and Jeanny Herault, "Blind separation of sources, Part I: An adaptive algorithm based on neuromimetic architecture", Signal Processing, 24, pp.1-10, 1991.

[7] P.Comon, C.Jutten and J.Herault, "Blind separation of sources, Part II: Problems statement", Signal Processing, 24, pp.11-20, 1991.

[8] E.Sorouchyari, "Blind separation of sources, Part III: Stability analysis", Signal Processing, 24, pp.21-29, 1991.

[9] S.Haykin, Adaptive Filter Theory, 3rd ed., Prentice-Hall, Inc. 1996. 
Table 1: Blind separation for asymmetrical distribution. Numerical data of $P$ are shown. P, U, NL and L mean Poisson, Uniform, Normal, Non-Limitation and Limitation of amplitude, respectively.

\begin{tabular}{|cc|cc|cc|}
\hline \multicolumn{2}{|c|}{ PU-NL } & \multicolumn{2}{|c|}{ PN-NL } & \multicolumn{2}{c|}{ PN-L } \\
\hline 0.998 & -0.064 & 0.902 & 0.739 & 1.001 & 0.022 \\
0.000 & 1.019 & -0.336 & 0.479 & -0.001 & 1.007 \\
\hline
\end{tabular}

Table 2: Blind separation for combination of voice and white noise. Numerical data of $P$ are shown. $\mathrm{L}, \mathrm{C}$ and $\mathrm{LC}$ means amplitude Limitation, Crosscorrelation and both are used for controlling learning process, respectively.

\begin{tabular}{|cc|cc|cc|}
\hline \multicolumn{2}{|c|}{$\mathrm{L}$} & \multicolumn{2}{c|}{$\mathrm{C}$} & \multicolumn{2}{c|}{ LC } \\
\hline 1.603 & 0.422 & 0.908 & 0.657 & 0.983 & 0.096 \\
-1.649 & 0.487 & -0.582 & 0.362 & 0.033 & 0.974 \\
\hline
\end{tabular}

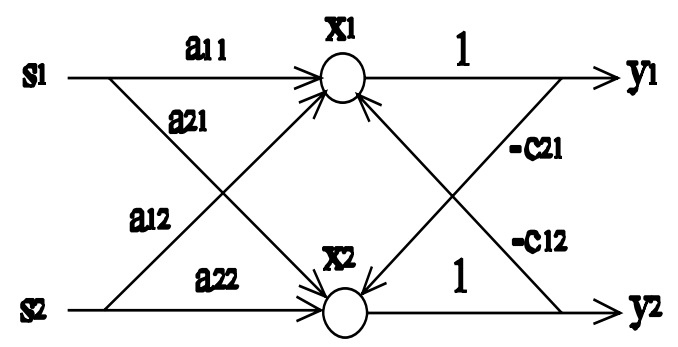

Figure 1: Block diagram of Jutten's blind separation.
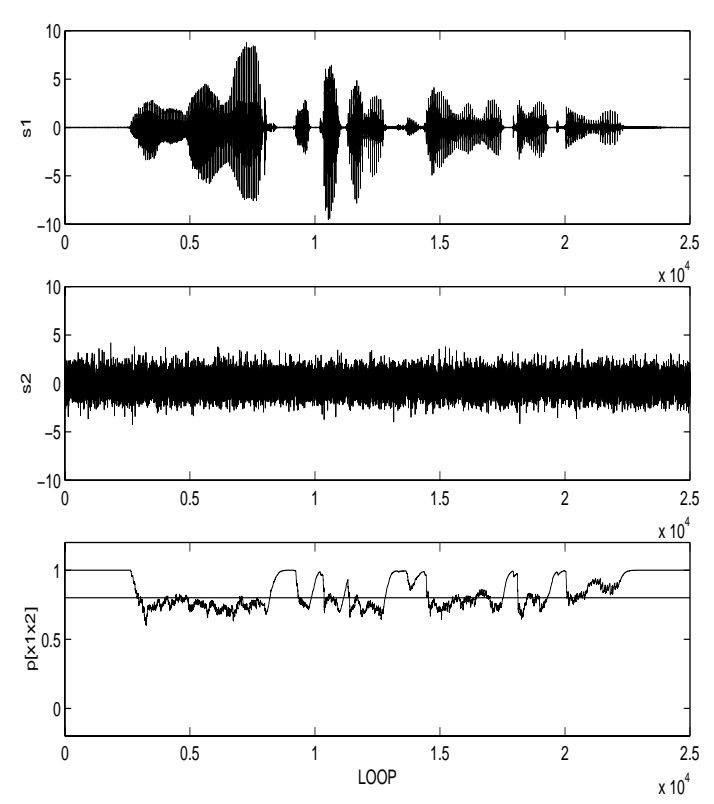

Figure 2: Waveform of voice and white noise, and cross-correlation $\rho_{x_{1} x_{2}}$.
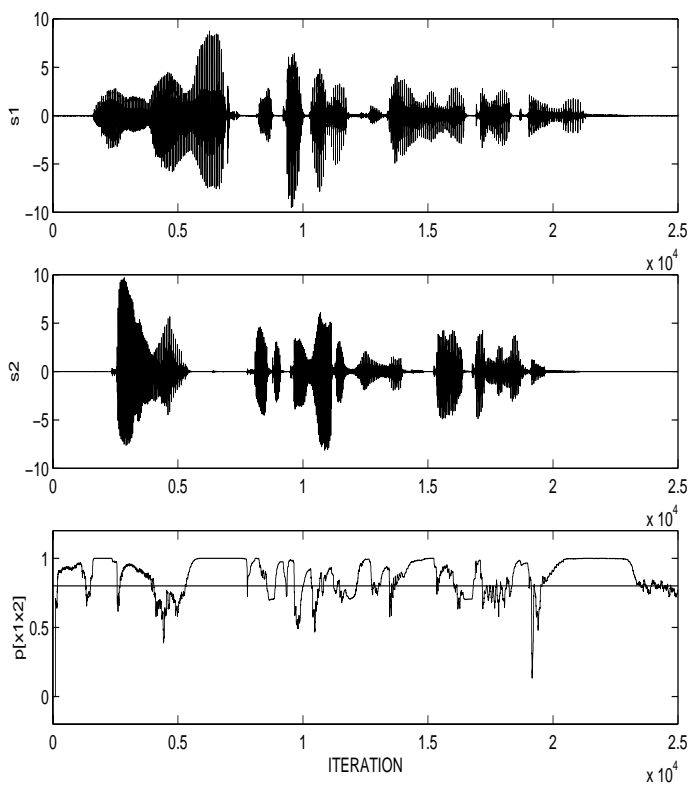

Figure 3: Waveform of voice of two men, and crosscorrelation $\rho_{x_{1} x_{2}}$. 